ARTICLE

DOI: $10.1038 / \mathrm{s} 41467-018-04230-8$

\title{
Molecular solar thermal energy storage in photoswitch oligomers increases energy densities and storage times
}

\author{
Mads Mansø $\varnothing^{1,2}$, Anne Ugleholdt Petersen', Zhihang Wang ${ }^{1}$, Paul Erhart (i) ${ }^{3}$, \\ Mogens Brøndsted Nielsen ${ }^{2} \&$ Kasper Moth-Poulsen (1) ${ }^{1}$
}

Molecular photoswitches can be used for solar thermal energy storage by photoisomerization into high-energy, meta-stable isomers; we present a molecular design strategy leading to photoswitches with high energy densities and long storage times. High measured energy densities of up to $559 \mathrm{~kJ} \mathrm{~kg}^{-1}$ ( $155 \mathrm{Wh} \mathrm{kg}^{-1}$ ), long storage lifetimes up to 48.5 days, and high quantum yields of conversion of up to $94 \%$ per subunit are demonstrated in norbornadiene/ quadricyclane (NBD/QC) photo-/thermoswitch couples incorporated into dimeric and trimeric structures. By changing the linker unit between the NBD units, we can at the same time fine-tune light-harvesting and energy densities of the dimers and trimers so that they exceed those of their monomeric analogs. These new oligomers thereby meet several of the criteria to be met for an optimum molecule to ultimately enter actual devices being able to undergo closed cycles of solar light-harvesting, energy storage, and heat release.

\footnotetext{
${ }^{1}$ Department of Chemistry and Chemical Engineering, Chalmers University of Technology, Kemivägen 10, 41296 Gothenburg, Sweden. ${ }^{2}$ Department of Chemistry, University of Copenhagen, Universitetsparken 5, 2100 Copenhagen $\varnothing$, Denmark. ${ }^{3}$ Department of Physics, Chalmers University of Technology, Kemivägen 10, 41296 Gothenburg, Sweden. Correspondence and requests for materials should be addressed to K.M.-P. (email: kasper.moth-poulsen@chalmers.se)
} 
S olar energy is a viable and inexhaustible source of energy for both electricity and heat production. In this context energy storage is a major challenge due to strong daily and seasonal variations in the availability of sunlight ${ }^{1}$. Molecular solar thermal (MOST) systems represent a promising avenue for harvesting and storing solar energy. In this approach a molecule is converted by photoisomerization into a higher-energy isomer, which is capable of storing the energy until released by a heat trigger or catalyst, converting the meta-stable isomer to the original light-harvesting isomer ${ }^{2-10}$. Norbornadienes have shown to be a promising candidate for MOST due to the high energy difference between the norbornadiene (NBD) and quadricyclane (QC) photoisomer of approximately $96 \mathrm{~kJ} \mathrm{~mol}^{-1}$ (Fig. 1), and the system has been shown to undergo heat-release by the action of cobalt-based catalysts $^{11-13}$. For these systems it has been previously shown that donor-acceptor substitutions provide an effective means for redshifting the longest-wavelength absorption, improving the solar spectrum match; compounds 1 and $\mathbf{2}$ present examples of such derivatives $^{14}$.

Two crucial challenges for a useful MOST system are the achievement of a sufficiently high energy storage density, ideally higher than $300 \mathrm{~kJ} \mathrm{~kg}^{-1}$ and light-harvesting in the visible region $^{15}$. Functionalization of the norbornadiene with donor and acceptor units has been used to tune absorption maxima, but this positive effect on solar absorption is counter-balanced by higher molecular weights, and hence lower energy densities ${ }^{11,16}$. Moreover, the positive effect on solar absorption typically also has a detrimental effect on the energy storage time, which is lowered when absorption is redshifted ${ }^{14}$. One possible solution to overcome this anti-correlation between redshifting and energy density that we present here is to couple one chromophore unit to several photoswitches. Here, in the context of NBD/QC systems, we find that it is attractive to form dimers or trimers, where the NBD units share the same donor and/or acceptor. Previously dimers of photoswitches such as dihydroazulenes ${ }^{17}$, dithienylethenes ${ }^{18,19}$, azobenzenes $^{20,21}$, and spiropyranes ${ }^{22,23}$ have been reported. Even other norbornadiene dimers, linked via phthalatamide bonds or conjugated ketones, have been prepared and studied, but showed very low quantum yields ${ }^{24,25}$.

The idea for the present work was to engineer the stability of the high energy photoisomer by having two electronically coupled photoswitches with separate barriers for thermal conversion (Fig. 2). The reasoning for this is that there is a blue shift after the first isomerisation (NBD-NBD to QC-NBD), leading to a higher energy of isomerisation of the second switching event (QC-NBD to QC-QC). Simultaneously having a shared donor reduces the molecular weight per norbornadiene unit. We chose to synthesize two series of dimers as well as one trimer of NBD that resemble the previously synthesized donor-acceptor monomers 1 and 2, with absorption onsets of 358 and $374 \mathrm{~nm}$, respectively ${ }^{14}$.

\section{Results}

Synthesis. The synthesis of NBD dimers and trimers is shown in Fig. 3 (all isolated as mixtures of diastereoisomers; only one of these is shown in each case). It was possible to obtain three alkyne-linked dimers as well as one trimer by Sonogashira couplings using the previously reported 2-chloro-3cyanonorbornadiene 3 as substrate ${ }^{14}$. The simplest dimer was synthesized by Sonogashira coupling with trimethylsilylacetylene, affording 4 in a yield of $63 \%$. The silyl protecting group was removed with potassium carbonate in $\mathrm{THF} / \mathrm{MeOH}$, and the resulting terminal alkyne was subjected to a second Sonogashira coupling with 3 to afford the acetylene-linked NBD dimer 5 in $47 \%$ yield. In the second Sonogashira reaction, standard conditions using phosphine ligands did not give any of the wanted products due to instability of the terminal acetylene. Instead, the $\mathrm{Pd}_{2} \mathrm{dba}_{3} / \mathrm{AsPh}_{3}$ catalyst system ${ }^{26}$ was used as it improved the reaction rate. Utilizing the same reaction conditions as for the synthesis of 4 with either 1,3- or 1,4-diethynylbenzene (6 or 7) gave the two NBD dimers 8 and 9 in 39 and $31 \%$ yield, respectively. Similarly, with 1,3,5-triethynylbenzene $\mathbf{1 0}$ as substrate, a triple Sonogashira coupling furnished 11 in a yield of $24 \%$.

To further investigate the effect of the spacer group, a second series with two NBD units directly linked to a phenylene spacer was synthesized (Fig. 4). Initial attempts employed 3 as substrate. Yet, a Suzuki cross-coupling with a double boronic acid did not yield any product (see Supplementary Figs. 6 and 8 for conditions). Instead, it was found that the desired product could easily be obtained from a Diels-Alder reaction between the known $^{27}$ para and meta bis(cyanoethynyl)benzenes 12 and 13, respectively, with cyclopentadiene (under microwave heating) affording the dimers $\mathbf{1 4}$ and $\mathbf{1 5}$ in yields of 77 and $45 \%$.

Spectroscopy. The new norbornadiene compounds were investigated with UV-Vis absorption spectroscopy, and the spectra are shown in Fig. 5. All of the spectroscopic studies were performed in cyclohexane as this solvent gave a larger spectral window for analysis of the switching events than toluene. As expected there is a large difference in the absorption onset and maxima between the meta and para isomers. The systems that are para substituted

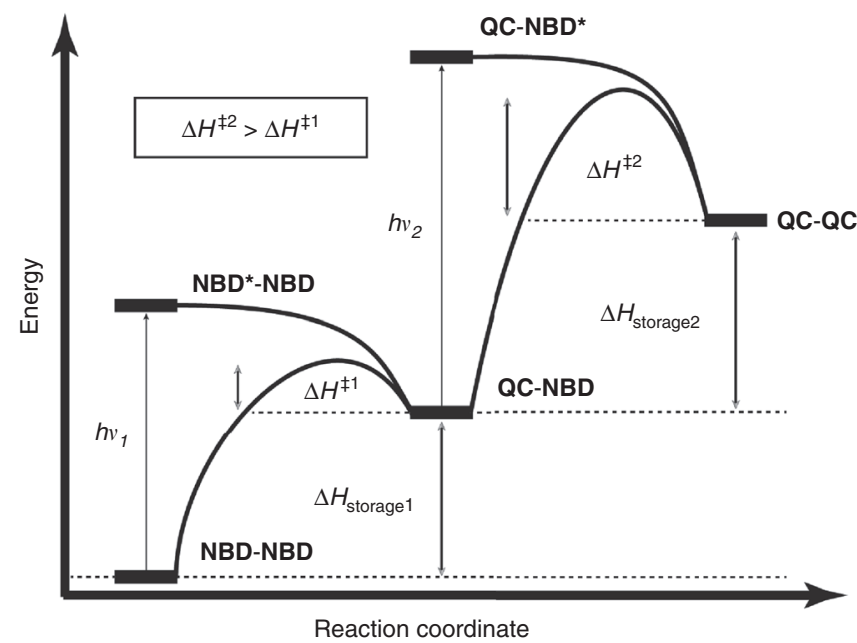

Fig. 2 Energy diagram. Schematic representation of the difference in halflife and irradiation energy for the two switching events

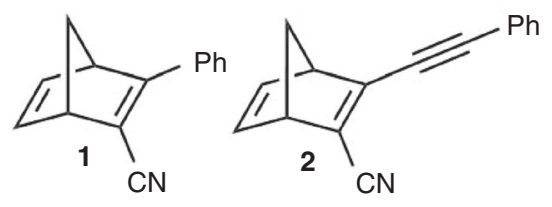

Fig. 1 Molecular system. The norbornadiene (NBD)-quadricyclane (QC) couple and the previously reported monomeric norbornadienes $\mathbf{1}$ and $\mathbf{2}$ 

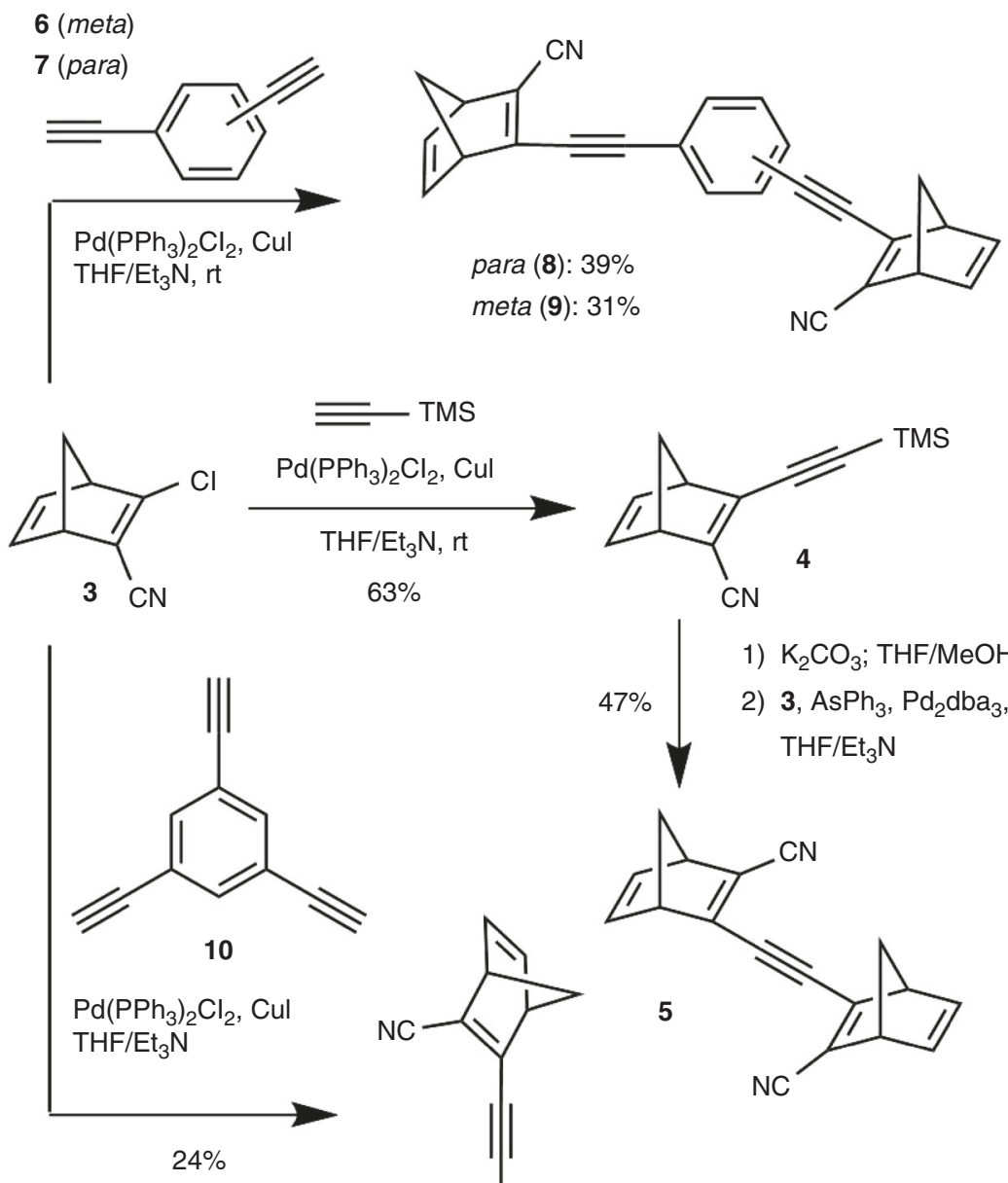

$63 \%$
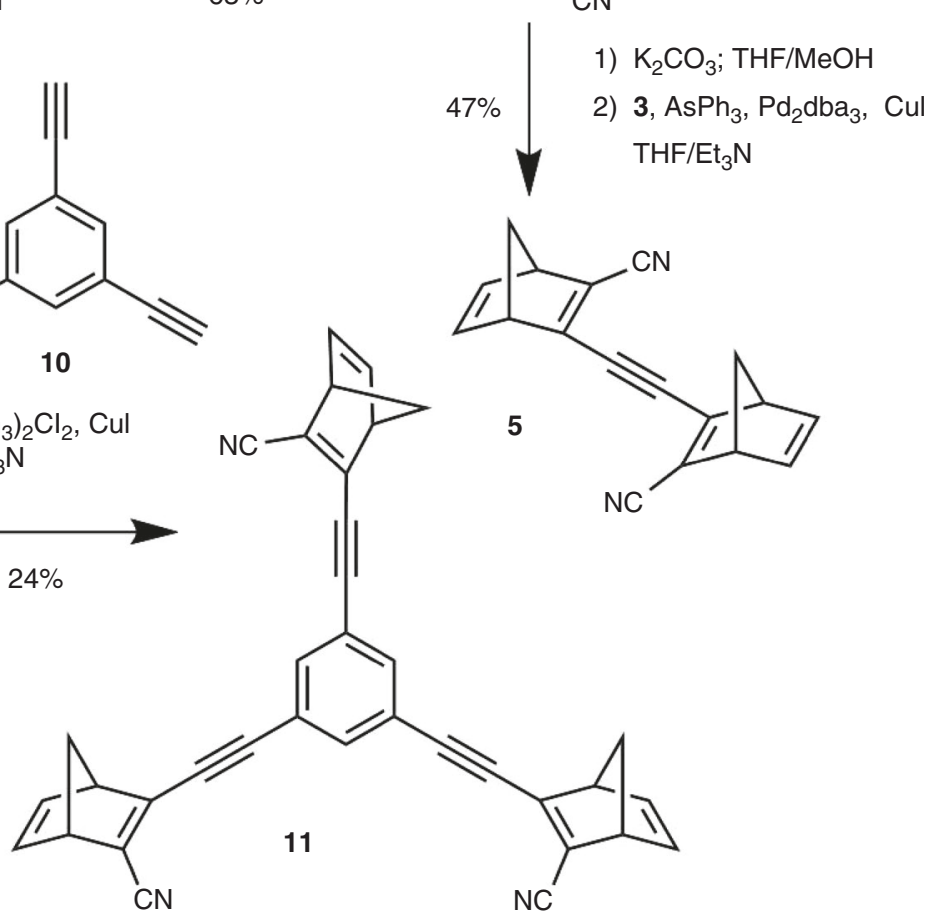

Fig. 3 Synthesis of alkyne-linked oligomers. Synthesis of acetylene-linked dimers 5, $\mathbf{8}$, and $\mathbf{9}$ as well as the trimer $\mathbf{1 1}$

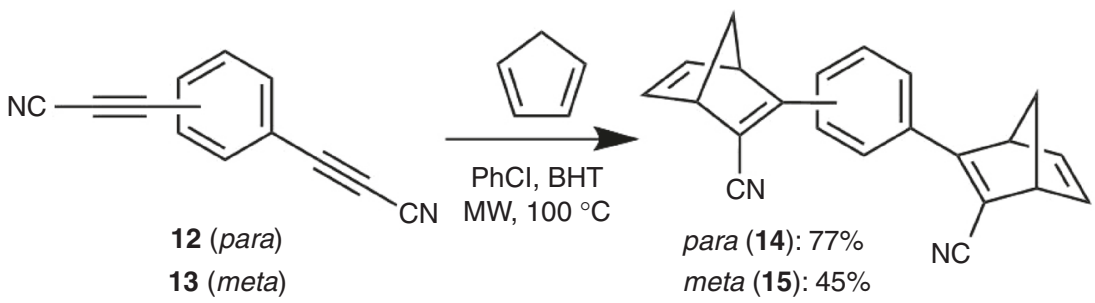

Fig. 4 Synthesis of phenylene-linked dimers. Synthesis of phenylene-linked norbornadiene dimers $\mathbf{1 4}$ and 15. BHT "butylated hydroxytoluene", i.e., 2,6-di (tert-butyl)-4-methylphenol; MW microwave heating

$(5,8$, and 14) give the highest onset of absorbance, which can be explained by the linear conjugation pathway between the two NBD chromophores, providing a larger conjugated system compared to the meta substituted compounds. The spectra of the meta substituted dimer 9 and the trimer 11 have very similar absorption onsets; yet, the trimer with three NBD units has a higher molar absorptivity. Interestingly the spectra are also similar to that of the corresponding monomer 2 (Fig. 5) in regard to longest-wavelength absorbance maxima and onset. This trend is also evident in the molar absorptivity, which for compound $\mathbf{2}$ is approximately half of the value for 9 . The same trend is seen for compounds 1 and 15, which have comparable absorption onsets and the longest-wavelength absorption maxima. The molar absorptivity of $\mathbf{1 5}$ is more than twice that of $\mathbf{1}$.

The next objective was to study the switching properties of the new NBD-NBD derivatives. Upon irradiation close to the 

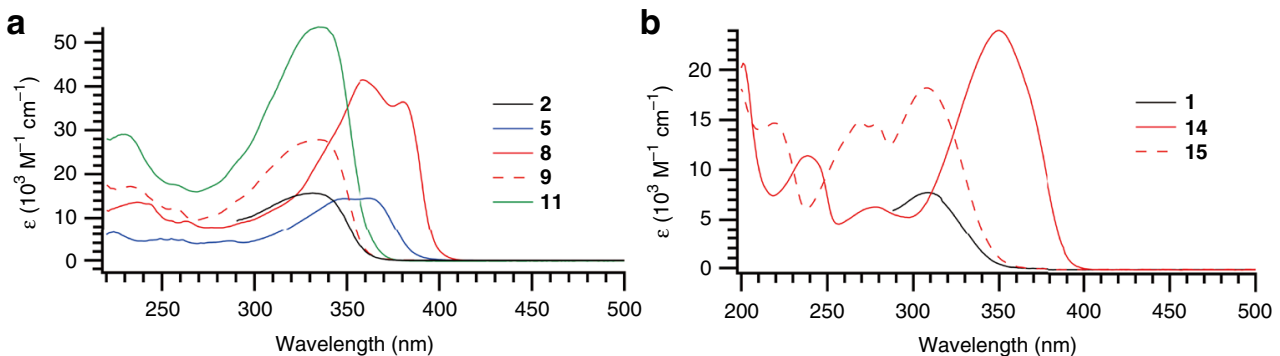

Fig. 5 Optical absorption spectra. a UV -Vis absorption spectra of acetylene-linked norbornadienes. Black line: $\mathbf{2}$, blue line: 5, red line: 8, red dashed line: $\mathbf{9}$ green line: 11. b UV-Vis absorption spectra of phenylene-linked norbornadienes. Black line: 1, red line: 14, red dashed line: 15. The spectra of monomers $\mathbf{1}$ and $\mathbf{2}$ were recorded in toluene

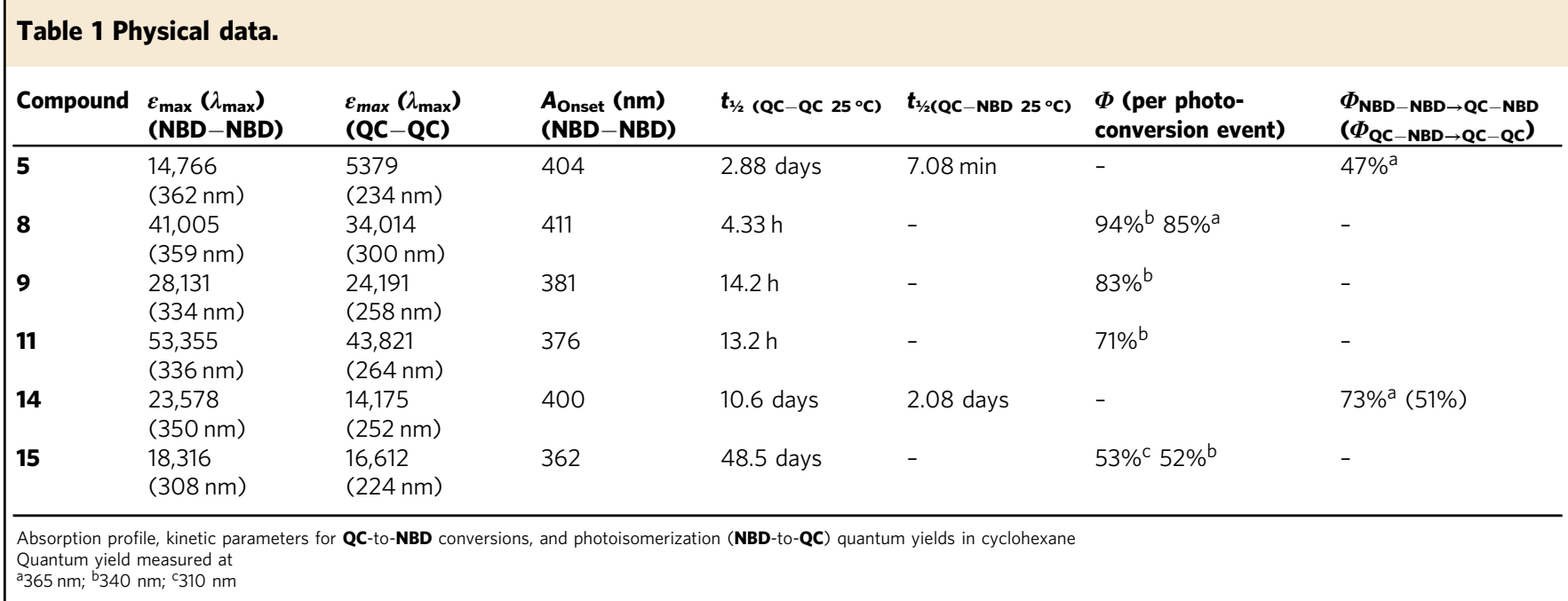

absorbance maxima (365 or $340 \mathrm{~nm}$ ), the dimers and trimer could all be fully converted to the corresponding QC-QC dimers, data shown in Table 1 . It was attempted to isomerize all of the norbornadiene dimers selectively to the intermediate QC -NBD form by irradiating around the onset of absorption. Switching to the QC-NBD could be achieved for compounds 5 and 14 by irradiation at $405 \mathrm{~nm}$. In addition the switching events were also studied by NMR spectroscopic analysis. For compound 5 it was found that irradiation at $405 \mathrm{~nm}$ forms a thermalphotostationary state between $\mathbf{5}_{\mathrm{NBD}-\mathbf{N B D}}$ and $\mathbf{5}_{\mathrm{QC}-\mathrm{NBD}}$, which is dependent on the intensity of the lamp and the temperature of the sample, due to fast back conversion of $\mathbf{5}_{\mathbf{Q C}-\mathbf{N B D}}$ (vide infra). The ratio found from NMR studies was 54:46 (see Supplementary Fig. 118). For 14, the isomerization to QC-NBD form could be obtained almost quantitatively by irradiation at $405 \mathrm{~nm}$, and the spectra of $14_{\mathrm{NBD}-\mathrm{NBD}}, 14_{\mathrm{QC}-\mathrm{NBD}}$ and $14_{\mathrm{QC}-\mathrm{QC}}$ are shown in Fig. 6. From NMR spectroscopic studies it was found that upon irradiation at $405 \mathrm{~nm}$, small amounts of $14_{\mathrm{QC}-\mathrm{QC}}$ were formed together with $14_{\mathrm{QC}-\mathrm{NBD}}$, and it was ultimately possible to convert the system to $14_{\mathrm{QC}-\mathrm{QC}}$ although this took over $40 \mathrm{~h}$ (see Supplementary Fig. 121 for details). Looking at the absorbance spectrum it is evident that even though it is possible to photoisomerize $14_{\mathrm{QC}-\mathrm{NBD}}$ at $405 \mathrm{~nm}$, the absorbance at $405 \mathrm{~nm}$ is close to non-existent, and thereby giving a spectral window for the sequential switching.

Quantum yields. Quantum yields of the photoisomerization events in cyclohexane were also measured for all the molecules using a reference procedure (Table 1$)^{28}$. For some of the molecules, when the absorbance profile allowed it, the quantum yield was measured at different wavelengths. This was done in order to independently probe the quantum yields of the two consecutive photoisomerization processes, ring closure of NBD-NBD and subsequently of QC-NBD (where their absorption profiles were taken into consideration). For compounds $\mathbf{8}, \mathbf{9}, \mathbf{1 1}$, and 15 the quantum yields are calculated based on the number of photoconversion events, taking into account that two or three NBD subunits are present per oligomer (see Table 1). The assumption is made that the units are behaving like independent NBD units and are having a similar absorption profile. For 14 and 5, which exhibit sequential switching, it was possible to measure the quantum yield for the NBD-NBD to QC-NBD process selectively. For $\mathbf{5}_{\mathrm{NBD}-\mathrm{NBD}}$ it was only possible to measure the quantum yield of the first photoconversion into intermediate $5_{\mathrm{QC}-\mathrm{NBD}}$ due to the fast rate of backreaction. In the case of $\mathbf{1 4}$ it is mainly the $\mathbf{1 4}_{\mathrm{NBD}-\mathrm{NBD}}$ form that absorbs, confirmed by the presence of an isosbestic point in the measurement, allowing the formation of $14_{\mathrm{QC}-\mathrm{QC}}$ to be neglected (see Supplementary Fig. 129). Furthermore, 14 could be irradiated at $405 \mathrm{~nm}$ to form $\mathbf{1 4}_{\mathrm{QC}-\mathbf{N B D}}$, and subsequently the quantum yield at $340 \mathrm{~nm}$ could be measured (see Table 1).

Kinetics. The kinetics of the QC-QC to NBD-NBD backreactions were determined at three different temperatures and the half-life at $25^{\circ} \mathrm{C}, t_{1 / 2}\left(25^{\circ} \mathrm{C}\right)$, was determined from an Arrhenius analysis (Table 1).

Interestingly, two markedly different rates of back conversion could be measured for both 5 and $\mathbf{1 4}$ (see Table 1). The half-life for the conversion of QC-NBD to NBD-NBD is remarkably faster than that for the backreaction of the fully isomerized 
a
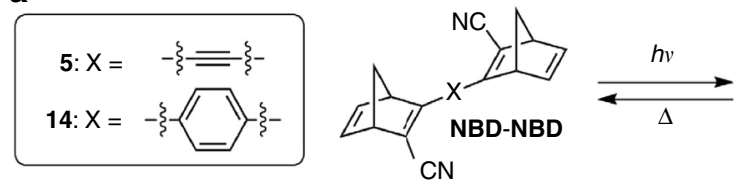

b

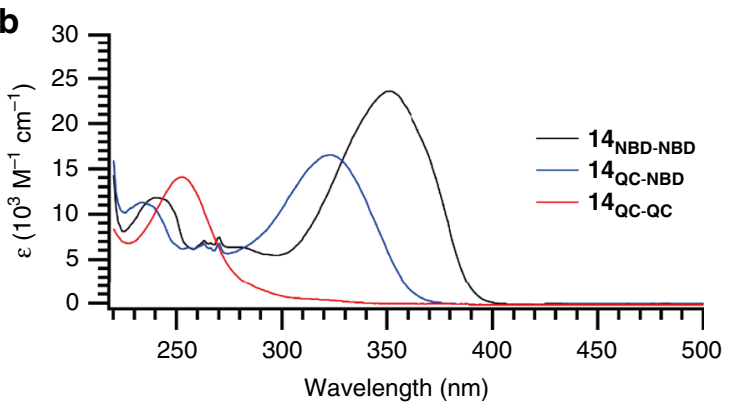

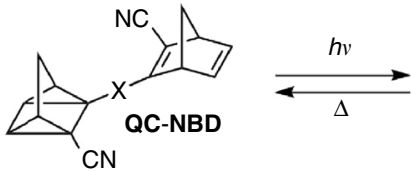

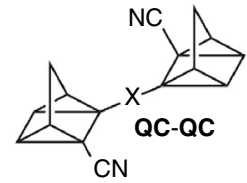

c

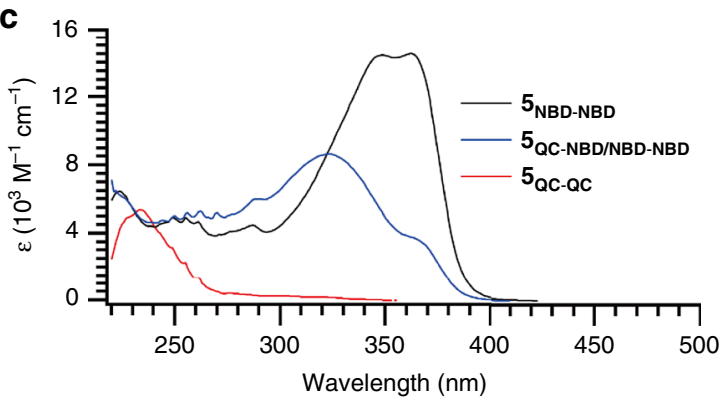

Fig. $\mathbf{6}$ Stepwise conversion of dimers. a Stepwise isomerization of $\mathbf{5}$ and $\mathbf{1 4}$ to the corresponding $\mathbf{Q C}-\mathbf{N B D}$ and $\mathbf{Q C}-\mathbf{Q C}$ isomers. $\mathbf{b}$ UV-Vis spectra of $\mathbf{1 4}$

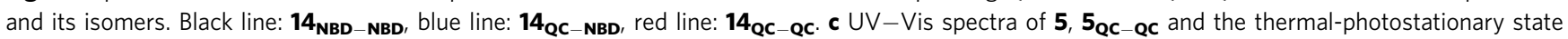
between $\mathbf{5}$ and $\mathbf{5}_{\mathbf{Q C}-\mathbf{N B D}}$. Black line: $\mathbf{5}_{\mathbf{N B D}-\mathbf{N B D}}$, blue line: the thermal-photostationary state between $\mathbf{5}$ and $\mathbf{5}_{\mathbf{Q C}-\mathbf{N B D}}$, red line: $\mathbf{5}_{\mathbf{Q C}-\mathbf{Q C}}$
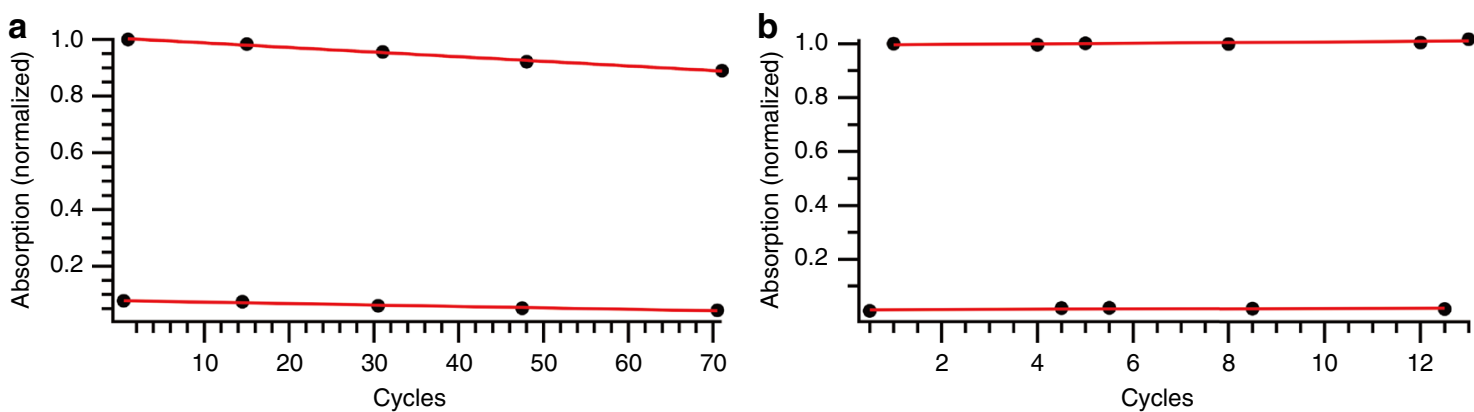

Fig. 7 Cyclability test. a Cyclability for $\mathbf{8}$ showing the normalized absorbance at $362 \mathrm{~nm}$ in cyclohexane at $50{ }^{\circ} \mathrm{C}$ irradiated at $365 \mathrm{~nm}$. b Cyclability for $\mathbf{1 4}$ showing the normalized absorbance at $350 \mathrm{~nm}$ in cyclohexane at $70^{\circ} \mathrm{C}$ irradiated at $340 \mathrm{~nm}$

QC-QC isomers. It was possible to fit the data for conversion of $\mathbf{5}_{\mathrm{QC}-\mathrm{QC}}$ and $14_{\mathrm{QC}-\mathrm{QC}}$ all the way back to the corresponding NBD-NBD isomers by single-exponential fits, due to the significantly faster conversion of QC-NBD to NBD-NBD. Fascinatingly, the first conversion from QC-QC to QC-NBD exhibits a higher barrier compared to the QC-NBD to NBD-NBD conversion, which we ascribe to the coupled nature of the two different electronic systems. For compounds 8, 9, 11, and 15 a discrimination of the different barriers could not be carried out due to similarities in the electronic properties of the QC-NBD and NBD-NBD forms of the molecules.

Cyclability. To investigate the stability over several cycles of photoisomerization followed by thermal back-reaction, a cycling test of 8 and $\mathbf{1 4}$ was performed (Fig. 7) by monitoring the UV -Vis absorption of the compounds at 362 and $350 \mathrm{~nm}$ respectively. The cyclability of 8 was tested over 71 cycles at $50{ }^{\circ} \mathrm{C}$ giving a decomposition of $0.16 \%$ per cycle, and for 14 the decomposition per cycle at $70{ }^{\circ} \mathrm{C}$ was found to be $0.11 \%$, showing that the decomposition for both these molecules is very small. This decomposition is shown as a decrease in the absorbance for $\mathbf{8}$, while for $\mathbf{1 4}$ there is an increase in the absorbance. This increase can be caused by evaporation of the solvent, or a decomposition product with a higher absorptivity. As some evaporation of the solvent could not be avoided, the cyclability of $\mathbf{1 4}$ is likely better than observed. Since the backreaction is relatively slow it was not possible to do the experiment at a lower temperature.
Energy densities. To obtain the energy densities of the highenergy QC isomers, the norbornadiene derivatives were irradiated at either 365 or $340 \mathrm{~nm}$ in $\mathrm{CDCl}_{3}\left(\mathrm{CDCl}_{3}\right.$ was selected due to the low solubility in cyclohexane), until fully converted; the solutions were concentrated by a stream of nitrogen and the resulting QC subjected to differential scanning calorimetry (DSC) (Table 2). Compounds 11 and 14 partly decomposed at the temperatures of the DSC experiment $\left(>140^{\circ} \mathrm{C}\right)$, so the energy densities of these compounds are not included. Isomerization of $\mathbf{5}$ in either $\mathrm{CDCl}_{3}$ or toluene- $d 8$ for DSC experiments $(\sim 1 \mathrm{M})$ could not be achieved by irradiation at either 365 or $340 \mathrm{~nm}$, possibly due to lower quantum yields in these solvents as well as the short lifetime of the QC-NBD form (minutes) making quantitative conversions impractical. The heat release data for QC-QC dimers of 8, 9, and 15 are listed in Table 2. For all of the molecules the heat release was also calculated using density functional theory (DFT) calculations (coordinates listed in Supplementary Data) ${ }^{19,20}$. The DSC sample for $\mathbf{9}$ was a mixture of $\mathbf{9}_{\mathrm{QC}-\mathrm{QC}}$ and $\mathbf{9}_{\mathrm{QC}-\mathrm{NBD}}$, which results in a large uncertainty, even though a correction taking this into account was performed. This is probably the reason for the large difference between calculated and measured values.

\section{Discussion}

In conclusion, we have developed efficient synthetic protocols for the preparation of high-performance norbornadiene dimers and trimers. The quantum yield of photoconversion is near quantitative, up to $94 \%$ per NBD subunit. For the para phenylene- 


\begin{tabular}{|c|c|c|c|c|}
\hline Compound & $\begin{array}{l}\Delta H \\
\left(\mathrm{~kJ} \mathrm{~mol}^{-1}\right)\end{array}$ & $\begin{array}{l}\Delta H \\
\left(\mathrm{~kJ} \mathrm{~kg}^{-1}\right)\end{array}$ & $\begin{array}{l}\Delta H_{\text {calc }} \\
\left(\mathrm{kJ} \mathrm{mol}^{-1}\right)\end{array}$ & $\begin{array}{l}\Delta H_{\text {calc }} \\
\left(\mathrm{kJ} \mathrm{kg}^{-1}\right)\end{array}$ \\
\hline 1 & 122 & 629 & 114.3 & 591.5 \\
\hline 2 & - & - & 118.5 & 545.9 \\
\hline 5 & - & - & 237.6 & 927.0 \\
\hline 8 & 183.3 & 514.2 & 231.1 & 648.5 \\
\hline 9 & 98.7 & 276.8 & 239.5 & 671.9 \\
\hline 11 & - & - & 362.4 & 731.3 \\
\hline 14 & - & - & 237.9 & 771.4 \\
\hline 15 & 172.5 & 559.3 & 232.0 & 752.3 \\
\hline
\end{tabular}

bridged NBD dimer 14, conversions between isomers occurred stepwise-both in the forward and backwards direction-and with significantly different NBD absorption maxima for the NBD-NBD and NBD-QC states. Our molecular design demonstrates the attractive feature of blueshifting the NBD absorption after the first NBD-to-QC photoisomerization as it ultimately furnishes a very energetic QC-QC state after the second photoisomerization as well as a prolonged storage time. As the first energy discharge (QC-QC to QC-NBD backreaction) is the rate-determining step, full discharge of the system will conveniently be promoted by triggering this event. The system contrasts in this respect related dihydroazulenevinylheptafulvene dimers incorporated in macrocyclic structures for which instead the first discharge is fast and the second slow ${ }^{29}$.

The calculated energy densities of the dimer and trimer systems of up to $927 \mathrm{~kJ} \mathrm{~kg}^{-1}\left(257 \mathrm{Wh} \mathrm{kg}^{-1}\right)$ and measured densities up to $559 \mathrm{~kJ} \mathrm{~kg}^{-1}\left(155 \mathrm{Wh} \mathrm{kg}^{-1}\right)$ greatly exceed the original targets of $300 \mathrm{~kJ} \mathrm{~kg}^{-115}$ highlighting the potential of applying molecular photoswitches in future solar thermal energy storage technologies. As the strongly coupled dimers $\mathbf{5}$ and $\mathbf{1 4}$ show a higher barrier for the first step in the thermal conversion compared to the second thermal conversion, this molecular motif can be used in future design of high-performance molecular switches for solar thermal energy storage as well as other applications. In future work a donor group could be attached to the benzene ring to give an enhanced redshifting of the absorbance spectra, or changing the benzene ring to more electron-rich aromatics. Another interesting approach would be to have two NBD units directly linked, in an attempt to increase the multimode switching by a greater coupling between the NBD subunits.

\footnotetext{
Methods

Materials. Cyclopentadiene was distilled by cracking dicyclopentadiene over iron filings and stored at $-80^{\circ} \mathrm{C}$, prior to use. Tetrahydrofuran used for Sonogashira coupling reactions was distilled over a sodium/benzophenone couple. All other chemicals were used as purchased from commercial sources. Purification of products was carried out by flash chromatography on silica gel $(40-63 \mu \mathrm{m}, 60 \AA)$. Thin-layer chromatography was carried out using aluminum sheets precoated with silica gel.
}

\footnotetext{
Measurements. Infrared (IR) spectra recorded on a Perkin-Elmer Frontier FT-IR instrument as films evaporated from $\mathrm{CDCl}_{3}$ onto an ATR attachment, where relative intensities are denoted as vw (very weak), w (weak), m (medium), s (strong), and sh (shoulder). All melting points and heat release of neat quadricyclanes were recorded on a Mettler Toledo DSC 2 apparatus. ${ }^{1} \mathrm{H}$ NMR (400 $\mathrm{MHz})$ and ${ }^{13} \mathrm{C}$ NMR $(100 \mathrm{MHz})$ spectra were recorded on a Varian $400 \mathrm{MHz}$ instrument or a Bruker $500 \mathrm{MHz}$ instrument with a noninverse cryoprobe using the residual solvent as the internal standard $\left(\mathrm{CDCl}_{3},{ }^{1} \mathrm{H} 7.26 \mathrm{ppm}\right.$ and ${ }^{13} \mathrm{C} 77.16$ ppm or $d 12$-cyclohexane, ${ }^{1} \mathrm{H} 1.38 \mathrm{ppm}$ and $\left.{ }^{13} \mathrm{C} 26.43 \mathrm{ppm}\right)$. All chemical shifts are quoted on the $\delta$ scale (ppm), and all coupling constants $(\mathrm{J})$ are expressed in $\mathrm{Hz}$. All solution-based spectroscopic measurements were performed in cyclohexane in a 1$\mathrm{cm}$ path length cuvette on either a Cary 60 or a Cary $100 \mathrm{UV}-\mathrm{Vis}$ spectrophotometer, scanning the wavelength from 600 to $200 \mathrm{~nm}$ coupled with Peltier temperature control. Photoswitching for wavelengths 310, 340, 365 and $405 \mathrm{~nm}$ were performed using Thorlabs LED lamps M310L3, M340L4, M365F1 and M405LP1 respectively. The thermal back reaction was performed by heating the
}

sample (cuvette) by a Peltier unit in the UV-Vis spectrophotometer. Quantum yields were measured by the published procedure ${ }^{28}$ using a high concentration regime (absorption above 2 at the irradiation wavelength) using potassium ferrioxalate and tris-phenanthroline iron (II) complex as a chemical actinometer and Thorlabs LED lamps M310L3, M340L4 and a fiber-coupled LED (M365F1) respectively for irradiation. Irradiation was done perpendicular to the cuvette in a fixed setup approximately $5 \mathrm{~cm}$ distance between LED and sample, with stirring, ensuring no movement of the lamp during the experiment. Mass spectra (HRMS) were either acquired by electron spray ionization (ESI) using an Agilent 1260 Infinity fitted with an Agilent 6120 quadrupole for or an ESP-MALDI-FT-ICR spectrometer equipped with a $7 \mathrm{~T}$ magnet (calibration of the instrument was done with NaTFA cluster ions). Elemental analyses were performed at London Metropolitan University. Compounds $3^{14}, \mathbf{1 2}^{27}$, and $13^{27}$ were made by their respective literature methods.

Computational methods. Calculations for the heat release from QC to NBD were performed at the B3LYP/6-311+ $\mathrm{G}^{*}$ level using the NWChem package ${ }^{30,31}$. All norbornadiene and quadricyclane compounds were fully relaxed. The storage energy was computed as the energy difference between the norbornadiene and quadricyclane forms.

Data availability. Data supporting this study can be found in the Supplementary Information file. Upon request to the corresponding author, further information supporting this study can be made available.

Received: 5 December 2017 Accepted: 10 April 2018

Published online: 16 May 2018

\section{References}

1. Gur, I., Sawyer, K. \& Prasher, R. Searching for a better thermal battery. Science 335, 1454-1455 (2012).

2. Kucharski, T. J., Tian, Y., Akbulatov, S. \& Boulatov, R. Chemical solutions for the closed-cycle storage of solar energy. Energy Environ. Sci. 4, 4449-4472 (2011).

3. Gurke, J., Quick, M., Ernsting, N. P. \& Hecht, S. Acid-catalysed thermal cycloreversion of a diarylethene: a potential way for triggered release of stored light energy. Chem. Commun. 53, 2150-2153 (2017).

4. Huang, J., Jiang, Y., Wang, J., Li, C. \& Luo, W. A high energy, reusable and daily-utilization molecular solar thermal conversion and storage material based on azobenzene/multi-walled carbon nanotubes hybrid. Thermochim. Acta 657, 163-169 (2017).

5. Brummel, O. et al. Photochemical energy storage and electrochemically triggered energy release in the norbornadiene-quadricyclane system: UV photochemistry and IR spectroelectrochemistry in a combined experiment. J. Phys. Chem. Lett. 8, 2819-2825 (2017).

6. Jorner, K. et al. Unraveling factors leading to efficient norbornadiene-quadricyclane molecular solar-thermal energy storage systems. J. Mater. Chem. A. 5, 12369-12378 (2017).

7. Masutani, K., Morikawa, M.-a \& Kimizuka, N. A liquid azobenzene derivative as a solvent-free solar thermal fuel. Chem. Commun. 50, 15803-15806 (2014)

8. Saydjari, A. K., Weis, P. \& Wu, S. Solar cells: spanning the solar spectrum: azopolymer solar thermal fuels for simultaneous UV and visible light storage. Adv. Energy Mater. 7, 1601622 (2017).

9. Wang, Z. et al. Evaluating dihydroazulene/vinylheptafulvene photoswitches for solar energy storage applications. ChemSusChem 10, 3049-3055 (2017).

10. Dreos, A. et al. Exploring the potential of a hybrid device combining solar water heating and molecular solar thermal energy storage. Energy Environ. Sci. 10, 728-734 (2017).

11. Lennartson, A., Roffey, A. \& Moth-Poulsen, K. Designing photoswitches for molecular solar thermal energy storage. Tetrahedron Lett. 56, 1457-1465 (2015).

12. Miki, S., Asako, Y., Morimoto, M. \& Ohno, T. Alumina-anchored cobalt porphine catalysts for the conversion of quadricyclane to norbornadiene. Bull. Chem. Soc. Jpn 61, 973-981 (1988).

13. Miki, S. et al. Alumina-anchored Cobalt(II) Schiff base catalyst for the isomerization of trimethyldicyanoquadricyclane to the norbornadiene. Chem. Lett. 17, 861-864 (1988).

14. Quant, M. et al. Low molecular weight norbornadiene derivatives for molecular solar-thermal energy storage. Chem. Eur. J. 22, 13265-13274 (2016).

15. Bren, V. A., Dubonosov, A. D., Minkin, V. I. \& Chernoivanov, V. A. Norbornadiene-quadricyclane-an effective molecular system for the storage of solar energy. Russ. Chem. Rev. 60, 451-469 (1991).

16. Gray, V., Lennartson, A., Ratanalert, P., Börjesson, K. \& Moth-Poulsen, K. Diaryl-substituted norbornadienes with red-shifted absorption for molecular solar thermal energy storage. Chem. Commun. 50, 5330-5332 (2014). 
17. Petersen, A. U. et al. Controlling two-step multimode switching of dihydroazulene photoswitches. Chem. Eur. J. 21, 3968-3977 (2015)

18. Uchida, K., Masuda, G., Aoi, Y., Nakayama, K. \& Irie, M. Synthesis of tetrathiafluvalene derivatives with photochromic diarylethene moieties. Chem. Lett. 28, 1071-1072 (1999).

19. Peters, A. \& Branda, N. R. Limited photochromism in covalently linked double 1,2-dithienylethenes. Adv. Mater. Opt. Electron. 10, 245-249 (2000)

20. Cisnetti, F. et al. Photochemical and electronic properties of conjugated Bis (azo) compounds: an experimental and computational study. Chem. Eur. J. 10, 2011-2021 (2004).

21. Robertus, J. et al. Kinetic analysis of the thermal isomerisation pathways in an asymmetric double azobenzene switch. Phys. Chem. Chem. Phys. 14, 4374-4382 (2012).

22. Fukushima, K., Vandenbos, A. J. \& Fujiwara, T. Spiropyran dimer toward photo-switchable molecular machine. Chem. Mater. 19, 644-646 (2007).

23. Ivashenko, O., van Herpt, J. T., Rudolf, P., Feringa, B. L. \& Browne, W. R. Oxidative electrochemical aryl C-C coupling of spiropyrans. Chem. Commun. 49, 6737-6739 (2013).

24. Bren, V. A. et al. Biphotochromic norbornadiene systems. Mol. Cryst. Liq. Cryst. Sci. Technol. Sect. A. 297, 247-253 (1997).

25. Yoshida, Z.-I. New molecular energy storage systems. J. Photochem. 29, 27-40 (1985).

26. Fouad, F. S., Crasto, C. F., Lin, Y. \& Jones, G. B. Photoactivated enediynes: targeted chimeras which undergo photo-Bergman cyclization. Tetrahedron Lett. 45, 7753-7756 (2004).

27. Sharma, K., Ram, S. \& Chandaka, N. Transition metal-free approach to propynenitriles and 3-chloropropenenitriles. Adv. Synth. Catal. 358, 894-899 (2016).

28. Stranius, K. \& Börjesson, K. Determining the photoisomerization quantum yield of photoswitchable molecules in solution and in the solid state. Sci. Rep. 7, 41145 (2017).

29. Vlasceanu, A. et al. Solar thermal energy storage in a photochromic macrocycle. Chem. Eur. J. 22, 10796-10800 (2016).

30. Kuisma, M. J., Lundin, A. M., Moth-Poulsen, K., Hyldgaard, P. \& Erhart, P. Comparative ab-initio study of substituted norbornadiene-quadricyclane compounds for solar thermal storage. J. Phys. Chem. C. 120, 3635-3645 (2016).

31. Valiev, M. et al. NWChem: a comprehensive and scalable open-source solution for large scale molecular simulations. Comput. Phys. Commun. 181, 1477-1489 (2010).

\section{Acknowledgements}

The Swedish Strategic Research Foundation and the Knut and Alice Wallenberg Foundation are acknowledged for financial support.

\section{Author contributions}

M.M. and A.U.P. carried out the synthesis and photophysical characterization, Z.W. and P.E. performed the calculations, M.B.N. and K.M.-P. conceived the idea. All authors contributed to writing of the manuscript.

\section{Additional information}

Supplementary Information accompanies this paper at https://doi.org/10.1038/s41467018-04230-8.

Competing interests: The authors declare no competing interests.

Reprints and permission information is available online at http://npg.nature.com/ reprintsandpermissions/

Publisher's note: Springer Nature remains neutral with regard to jurisdictional claims in published maps and institutional affiliations.

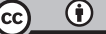

Open Access This article is licensed under a Creative Commons Attribution 4.0 International License, which permits use, sharing, adaptation, distribution and reproduction in any medium or format, as long as you give appropriate credit to the original author(s) and the source, provide a link to the Creative Commons license, and indicate if changes were made. The images or other third party material in this article are included in the article's Creative Commons license, unless indicated otherwise in a credit line to the material. If material is not included in the article's Creative Commons license and your intended use is not permitted by statutory regulation or exceeds the permitted use, you will need to obtain permission directly from the copyright holder. To view a copy of this license, visit http://creativecommons.org/ licenses/by/4.0/

(C) The Author(s) 2018 